\title{
Italian Language, its Position in the European Languages, its Relation with the Past, Present and Future, its Linguistic Values, Story and Potential
}

\author{
Arben Skendaj Ph.D \\ University of Tirana, Faculty of Foreign Languages \\ Department of Italian Language \\ Email: arskendaj@hotmail.com \\ Sofia Delijorgji Ph.D \\ University of Tirana, Faculty of Foreign Languages \\ Department of Slavic and Balkan Languages \\ Email; paskosofia@yahoo.com
}

\section{Doi:10.5901/mjss.2013.v4n3p39}

\begin{abstract}
Italian is a neolatin language, which derives, like many other languages in Europe, by Latin. But it has an original and totally different story from the others. In its centennial road, it has found its definite and dignified evaluation after many difficulties and hassles. Differently from the rest of other European languages situation, Italian is ranked last, but this is not a reason to consider it last in terms of value and linguistic capacity. We can see that linguistic aspects, phonetics, morphology, syntax and lexicology of Italian have and advantage in these sectors, which shows solidity and a kind of "supremacy" nowhere else to be found. This is an assertation that has been proved to be true in the course of time. I think that Italian nowadays and also in the future will have a paramount linguistic position, and that not for sentimental or delusional reasons, but for well-grounded arguments. Neolatin languages are almost completely of Latin origin. Italian, living and operating in the same territory where Latin once operated has cultivated itself with Latin.
\end{abstract}

Keywords: language, vocabulary, etymology, semantics, loanwords

L'italiano fa parte della famiglia delle lingue neolatine insieme al francese, spagnolo, portoghese e romeno. Ma la specificità dell'italiano rispetto agli altri membri della famiglia, credo sia il fatto che l'italiano lo considero più che una lingua neolatina. L'italiano si parla nello stesso territorio dove si è parlato il latino e questo non potrebbe considerarsi irrilevante, molti linguisti lo hanno considerato solo neolatina. Volendo fare un paragone con altre due lingue che sono dirette discendenti dalla loro variante antica che sono greco antico, greco moderno, turco antico, turco moderno che certamente sono collegate talmente da considerarsi quasi la stessa lingua, seppur con profonde trasformazioni e differenze che qualcuno ha azzardato l'idea che si tratti addirittura di lingue viste come parenti alla lontana. Per cui credo che l'italiano abbia più di un semplice rapporto di origine neolatina col latino, esso può essere visto in un rapporto simile al rapporto delle precedenti lingue succitate, greco antico e moderno e lo stesso del turco antico e moderno. Cioè come fosse latino antico e latino moderno inteso l'italiano si intende. Vedendo anche poi tecnicamente, sotto vari aspetti grammaticali e lessicali, l'italiano lascia l'impressione che sia la più fedele al latino, la sua evoluzione nel tempo sembra sia stata una trasformazione più naturale rispetto alle altre lingue neolatine che appaiono più delle discendenze trapiantate di quello che è l'italiano in rapporto col latino. Questo certamente senza che alluda a qualche valutazione soggettiva di sopravalutazione o sottovalutazione verso nessuna lingua. Ma semplicemente per precisare che l'italiano ha un privilegio di rispecchiare più originalmente e fedelmente il latino magari un po' più delle restanti lingue neolatine.

II latino era la lingua del più grande impero di tutti i tempi, dell'Impero Romano. Come sappiamo a quel tempo questo impero ha conosciuto degli altissimi livelli di sviluppo sociale, culturale, politico, giuridico, medico, tecnologico, linguistico, letterario ecc. E questi sviluppi certamente dovevano materializzarsi anche nel linguaggio e verifichiamo così un lessico molto sofisticato e che oggi questi termini fanno da monopolio anche dopo 2000 anni in tutte le lingue moderne europee e non, e che per di più si parlano a livello globale. Questi "patrimoni" lessico-semantici per molte lingue (il riferimento al ceppo italo-latino) resta ancor oggi un indiscusso e comodissimo ripiego, "salvagente", che risultano sempre essere trapianti lessicali dignitosi per molte lingue, incluso l'inglese, per di più lingua di ceppo anglo-germanico. $\mathrm{E}$ di esempi ne possiamo citare moltissimi qui, ma non è quello lo scopo, forse in altra sede si potrebbe anche fare. 
Ma se guardiamo le lingue europee, appartando il lessico di base (riferito alle lingue anglo-sassoni) noi identifichiamo uno strato lessicale ormai naturalizzato in queste lingue che rispecchia un'enorme fetta nel lessico di tali lingue. E' una percentuale, che supera di gran lunga la metà del lessico in globale di suddette lingue.

Questi scambi non presentano, non suggeriscono alcun complesso o handicapp per queste lingue, e in queste situazioni di prendere prestiti non si salva nessuna lingua, senza escludere anche l'albanese di cui parlerò verso la fine della relazione. Ma la ragione per cui si prendono questi prestiti è la necessità di rispecchiare linguisticamente i nuovi concetti, fenomeni o cose, che si inventano e che per farlo quasi sempre, per le lingue suddette, la via di rivolgersi alle lingue classiche è la soluzione più riuscita e immune dal rischio del ridicolo, cioè rimodellando modelli classici latini e anche greci sembra la soluzione più fortunata dal punto di vista linguistico. Così mi viene in mente un esempio tipico senza che si pensi di esaurire l'argomento, ma solo per rendere l'idea. La parola computer è entrata preponderatamente se non "prepotentemente" in tutte le lingue del mondo, la storia di questa parola risale più o meno agli anni 60 quando fu inventato il primo computer in California, U.S.A.. Nessuno avrebbe pensato che dopo questo strumento di lavoro sarebbe diventato così diffuso e utile in tutti gli angoli più disparati della terra. A prima vista il suffisso nominale -er, tipico dell'inglese sembra, che suggerisca un'origine anglossassone per questa parola, ma ci si sbaglia perché questi meccanismi modificatori morfo-lessicali funzionano tranquillamente in ogni lingua e si aggiungono come prefisso 0 suffisso a una base straniera lessicale adattandola così alla struttura morfo-lessicale della lingua, che l'accoglie e questo succede spesso, anche dal punto di vista fonetico. Questa parola deriva dal latino e oggi l'italiano computo che vuol dire calcolo, conto, perché il computer inizialmente è nato appunto per fare calcoli, e da qui oggi in italiano sopravvive una nutrita famiglia del computo; computare che vuol dire fare calcoli e il nome computazione, computazionale (agg.), computatore (sost.).

La lingua italiana ha avuto un percorso diverso in seno alle altre lingue europee, la sua storia è per certi versi particolare, non che le altre non ce l'abbiano particolare. "Quando il linguista descrive una lingua, ad esempio, l'italiano, costruisce ciò che gli scienziati di solito definiscono un modello"( John Lyons "Manuale di Semantica", Laterza, Bari 1980 p. 29.). La storia di una lingua non si può staccare dalla storia di una nazione di un popolo, essa è strettamente collegata, se non condizionata in gran parte da essa. La storia dello Stato italiano, relativamente recente, di un secolo e mezzo circa ha condizionato anche lo sviluppo della lingua italiana. L'italiano si è dimenato nei secoli senza che avesse una sua configurazione e fisionomia stabile e permanente paragonato alle altre lingue europee. L'inglese, il francese, lo spagnolo, il portoghese, il tedesco ecc si sono standardizzate e configurate molti secoli prima dell'italiano. Viene da chiedersi com'è stato possibile questo, se il contesto e l'ambiente socio-politico dove sono esistite queste lingue è stato simile se non identico. Bisogna riconoscere, che il motivo non può essere uno solo, ma tanti e spesso molto complicati da analizzare e di natura tutt'altro che linguistica. Nei paesi rispettivi alle lingue sopra citate, esclusa l'talia, hanno avuto una storia diversa da quella italiana. E' stata proprio la situazione storico-politica a contribuire a standardizzare il modello di queste lingue. La storia di questi paesi si è svolta avendo al centro dell'attenzione una figura politica, unitaria a livello nazionale, oltre che ad avere anche figure del potere ecclesiastico, la figura di un re, di un monarca è stata imposta come un riferimento di autorità indiscussa e quasi autoritaria derivato dal potere assoluto, per cui anche il modello linguistico standard a livello nazionale queste popolazioni se lo sono dovuti accettare e adottare senza avere il dubbio di qualche alternativa facoltativa.

La soluzione del modello linguistico standard a livello nazionale veniva imposto dalla situazione politica. Certamente anche i dialetti saranno sopravissuti in queste realtà europee, ma hanno avuto sicuramente una condizione di sottordine in confronto al modello standard nazionale.

La situazione in Italia è completamente diversa, come per raccontare una storia tutta sua. L'Italia come sappiamo per tanti secoli è stata divisa in Stati e staterelli, senza ignorare qui l'esistenza di qualche saltuario e provvisorio regno 0 repubblica solo a livello regionale. Avendo questa situazione politica e sociale, anche di qui si capisce che la necessità di comunicare entro i confini nazionali non c'era, un'autorità geograficamente nazionale mancava e per giunta nessuna pressione veniva esercitata per fare questa unione linguistica. In questo contesto le lingue locali e i dialetti avevano tutto il sacrosanto diritto di fiorire e pullulare. L'unica autorità, l'unico riferimento a livello nazionale era la figura del papa che certamente apparteneva al potere ecclesiastico. Ma questa figura non poteva assolutamente adempiere al ruolo eseguito dai re nei diversi paesi, questa funzione il papa non poteva svolgerla. Avrà magari servito a causare altri effetti collaterali a livello sociale e politico anche positivi, ma quello linguistico non lo poteva fare, seppure il periodo di tempo di autorità religiosa è stato forse anche più lungo di più del regno più lungo mai esistito .

Di qui ovviamente è facilmente deducibile che questa popolazione si sia risolta i suoi problemi di comunicazione arrangiandosi col dialetto locale, regionale senza dover far i conti con un modello nazionale standard, come viene concepito oggi geograficamente. I dialetti italiani che corrispondono in via di massima oggi sono più o meno alle zone $\mathrm{e}$ 
regioni, che era una volta la divisione medioevale regionale. Bisogna sottolineare qui che qualche dialetto è più stretto di qualche altro, considerando qui anche le estremità geografiche, per esempio un piemontese 0 veneto ha più fatica a capirsi con un siciliano.

Per corroborare questidea la storia ci viene incontro, suggerendo che Napoleone al tempo dell'occupazione in Italia si sia stupefatto di come ufficiali italiani provenienti da diverse regioni italiane parlassero diverse lingue e non una lingua sola, questo perché naturalmente in Francia questo era una cosa strana, una lingua standard in Francia era già un fatto compiuto da molto tempo. E in fatto di modello linguistico standard, io direi, che presenterebbe molti aspetti positivi dal punto di vista sociale, perché aiuterebbe a diffondere una mentalità di gruppo molto più velocemente, a emancipare molto più presto, a civilizzare molto più rapidamente.

$\mathrm{Ma}$ in Italia a un modello standard già ci aveva pensato Dante Alighieri nel medioevo, suggerendo anche tecnicamente il dialetto da proporlo come tale. Ma era prematuro, perché le condizioni socio-politiche non lo favorivano questo passo. E questo in Italia non avvenne neanche subito dopo la riunificazione politica dell'Italia, ma ci è voluto l'avvento dei massmedia negli anni 60, e come Tullio De Mauro ha " evidenziato con precise argomentazioni, il ruolo svolto dalla radio e - a partire dal 1954 - dalla televisione, come fonti di lingua, tendenti a imporre latamente modelli unitari" ( Tullio De Mauro "Storia linguistica dell'Italia unita", Laterza, Bari 2008, p. 125-26. ), cioè a diffondere un modello standard per l'inevitabile necessità di comunicare a livello nazionale, perché erano appunto ormai proprio le condizioni socio-politiche a imporlo come bisogno. "L'italianizzazione dei dialetti e quindi il diffondersi dell'italiano in tutti gli ambiti della società è un evento cruciale della nostra storia recente" (Luca Serianni e Pietro Trifone "Storia della lingua italiana", p. 371. Einaudi Editore Torino 1993.)

E il dialetto che si preferì come modello da proporre questa volta la storia diede ragione a Dante; fu scelto il modello toscano. Comunicare diventò necessità nazionale, e il modello standard si avviò senza traumi e difficoltà, questo processo fu fluido e diffuso in tutta l'Italia.

Scrivere, leggere in Italia diventò una norma chiarissima e indiscussa. Così giornali, letteratura, testi scolastici e universitari si uniformano tutti linguisticamente in un modello quasi perfetto: "...dato il profondo rivolgimento delle abitudini linguistiche e la forte espansione della lingua standard in tutti gli ambiti d'impiego sotto la spinta dei mutamenti sociali, economici, culturali del nostro secolo" (P.Benigna, M.Berretta, M. Dardano, E.Magno Caldognetto, A. M. Mioni, B. Mortara Garavelli, P. Ramat, R.Simone, A.Sobrero, "Introduzione all'italiano contemporaneo. Le strutture. Editori Laterza 1993. p. 324 ).

Verso gli anni 90-2005 credo di avere notato una tendenza dei media sotto l'influenza dei materiali tradotti da lingue di ceppi diversi tipo anglo-germanico, specie il parlato, di sostituire delle forme verbali, esattamente tempi di verbi come passato remoto, il congiuntivo e il condizionale con tempi di italiano dell'indicativo come imperfetto, passato prossimo sovraccaricandoli di usi eccessivi provocando spesso equivoci indesiderati, inevitabili.

I dialetti italiani sono sopravissuti e alcuni di questi sono dei veri pilastri nel tempio della lingua italiana. Volendo tracciare un quadro completo dell'italiano vorrei far rifletter su un fatto ancora curioso. Come sappiamo il lessico dell'italiano si presenta oggi, agli occhi degli italiani e degli stranieri come un'entità lessicale di circa 130.000 voci cifra che più 0 meno vantano egregiamente anche altre lingue europee che ovviamente riferita nei grandi dizionari monovolumi commerciabili oggi in Italia. Perché in effetti " la nostra lingua possederebbe tra i 300.000 e 500 mila vocaboli ( di questi 100.00 sarebbero di quelli di uso non specialistico)" (P.Benigna, M.Berretta, M. Dardano, E.Magno Caldognetto, A. M. Mioni, B. Mortara Garavelli, P. Ramat, R.Simone, A.Sobrero, "Introduzione all'italiano contemporaneo. Le strutture. Editori Laterza 1993. P.296 ). Lessicalmente, semanticamente, linguisticamente risulta che il lessico di una lingua, giustamente, va rispecchiato tutto, nelle forme rappresentative, ma quello che si usa normalmente e anzi è un obbligo che unità morfo-lessicali usate nello scritto e nel parlato vanno registrate nei dizionari seri di consultazione. $\mathrm{E}$ questa è una regola d'oro, perché i lessicografi sono interessati a inserire tutte le parole usate nella lingua, anzi più ne mettono più ne vantano una grande cifra e completezza di lessico, per cui giustamente non vogliono tralasciare niente. "Nell'ambito della grammatica italiana, considerata "difficile" (vale a dire ricca di forme, non distribuite equamente, e di ridondanze), si sottolinea tra l'altro la complessità: 1) del sistema flessionale..." ( Luca Serianni e Pietro Trifone "Storia della lingua italiana", p. 384. Einaudi Editore Torino 1993) . In italiano ci sono due categorie morfo-lessicali a pieno diritto linguistico, ma che nei dizionari non vengono registrati, fatto molto strano per una lingua, che non figuri le sue unità lessicali. Queste due forme lessicali, in origine morfologiche sono potenzialmente utilizzabili, ma lessicograficamente latenti. Esse sono unità realmente, degnamente lessicali e sono il participio presente e gli alterati. Guardando numericamente queste unità risulterebbe un numero molto elevato, che supererebbe decine di migliaia di voci. Per carità parte minima di queste unità figura già nei dizionari, ma il grosso no. Molti participi presenti per necessità lessicale si sono aggettivati o sostantivati, ma la maggior parte non ha preso ancora la "cittadinanza" lessicale, cioè non sono 
vocabolarizzati, ma che nel parlato e nello scritto però tutti gli infiniti per motivi lessicali, che sappiamo, possono essere utilizzati come participi presenti.

Anche se bisogna ammettere che questo processo, o movimento nasca come fenomeno di natura morfologica, però come uso e necessità è un purissimo fenomeno di lessicologia. II participio presente è un tipico aspetto di lingua latina arrivato fino ai nostri giorni così fiorente in italiano e per giunta esportato addirittura, molte volte, anche in altre lingue sotto questa veste. Come sappiamo il participio presente equivale alla congiunzione "che più lo stesso verbo al presente indicativo terza persona singolare o plurale", dipende dal contesto, per esempio ignorante equivale che ignora, simpatizzante che simpatizza ecc. Ma migliorare non suggerisce nessun vocabolario che esista come unità lessicale a parte, ma nessuna grammatica e semantica impedisce di usare il participio presente del verbo migliorare per necessità momentanea del parlato o dello scritto per esempio: "Queste sono le ultime tecnologie miglioranti la qualità dei prodotti", questo è italiano perfetto, ma nei vocabolari non si trova mai questa unità lessicale e tantissime altre simili. Comunque un motivo c'è per cui si giustifica l'uso del participio presente che è quello della concisione, però non si salva dal fatto che è una forma più contorta rispetto alla quasi frase congiuntiva; così miglioranti è uguale a "che migliorano". Della maggior parte degli infiniti in italiano non troveremo nei dizionari questi participi presenti, quelli trovati sono ormai definitivamente lessicalizzati senza escludere un loro parallelo uso grammaticale originario, che è quello della frase subordinata congiuntiva. Queste unità lessicali in partenza sono stati dei semplici tentativi di usi verbali e grammaticali ( come alternativa della frase congiuntiva), ma solo dopo si sono, per necessità, lessicalizzati e se oso inventare un neologismo "semanticizzati" e sono diventate delle vere serie parole di lessico italiano ( usate come aggettivi e sostantivi), senza però perdere l'originario loro uso grammticale a seconda della necessità.

In linea di massima così funziona anche per gli alterati considerando tutto il corpus dei nomi e che per un nome per esempio a volte ci sono anche 5-6 alterati senza dimenticare qui che di alterati ce n'è anche di aggettivi e verbi. Non credo personalmente che situazioni così sofisticate e potenziali possano avere e gestire altre lingue. lo questi aspetti dell'italiano li interpreto come mezzi supplementari, enormi di arricchimento del lessico e questo non fa che suggerire, che l'italiano in fatto di problemi e necessità lessicali sarebbe l'ultima lingua, al mondo, che ne risentirebbe.

Come albanesi credo sia doveroso fare un bilancio di scambi lessicali, linguistici con l'italiano e non perché è il caso di parlare dell'italiano. L'albanese è una lingua, che appartiene al ceppo delle lingue indoeuropee e senza ramificazioni. Come sappiamo a stabilire questo, cioè l'appartenenza a una famiglia linguistica è la grammatica e il lessico di base di cui l'albanese si distingue come originale.

Ma oltre al lessico di base in albanese il resto del lessico è di origine italo-latina. Questo lessico costituisce il lessico medio e medio alto dell'albanese e di ogni lingua che praticamente appartiene agli strati sociali elevati socialmente e culturalmente. L'albanese queste esigenze lessicali non ha la tendenza di risolverle da lingue più vicine, forse, geograficamente per esempio dal greco, turco, lingue slave ecc, ma senza dubbio si rivolge all'italiano per risolvere problemi lessicali, dovendo riconoscere un debito lessicale enorme se di "debito" possiamo parlare.

Statisticamente poi sopratutto gli ultimi 20 anni con la libertà di stampa c'è stato un aumento numerico lessicale dall'italiano ed è una tendenza, ma non è un compito assolutamente facile come risulta dalla realtà. II fatto che l'albanese preferisca adottare prestiti dall'italiano, più che da altre lingue credo che sia per motivi prevalentemente di natura fonica, fonetica e lessicale perché come sappiamo il primo impatto con una parola straniera è l'aspetto fonetico che colpisce e l'italiano pare più digeribile per la fonetica albanese. Un secondo motivo sarebbe quello lessicale perché l'italiano oggi dal punto di vista linguistico si presenta come una "supremazia" linguistica a livello mondiale. Il discorso del lessico medio e medio alto che dicevamo prima è esattamente questa fascia di lessico, che è entrata nelle lingue non neolatine e come dicevamo supera la metà del lessico di queste lingue. A vedere bene questo lessico ha avuto tutto il tempo necessario per stabilizzarsi e convivere col resto del lessico della lingua prestante queste parole. Ma queste parole hanno avuto un trapianto ponderato, sereno e non traumatico come lo ha vissuto l'albanese. In queste lingue oltre a trovare le stesse parole neolatine troviamo anche una quasi perfetta corrispondenza semantica addirittura polisemica in queste parole insomma, hanno mantenuto la stessa semantica dell'originale. Ho l'impressione, che in albanese non si ha sempre la preoccupazione di mantenere la motivata e naturale semanticità delle parole prestate come se si avesse semplicemente bisogno di combinazioni fonetiche certificate, ma che poi del significato originale non se freghi poi tanto. Sembra che si noti la tendenza di buttare troppa carne al fuoco, che poi se "brucia o meno si vede nel tempo". Ma questa troppa fretta e nessuna cautela non salverebbe da situazioni ridicole e di poca serietà dal punto di vista lessico-semantico, ma essendo un fatto di impronunciata responsabilità il caos è legale.

Tornando al lessico medio e medio alto neolatino, in conclusione direi che questo lessico sia comune a tutte le lingue di società più civilizzate e che imparando il lessico dell'italiano io credo che uno riesca ad avere una chiave per aprire molte porte linguistiche, culturali. Volendo raffigurarlo questo lessico medio e medio alto potremo concepirlo come 
cerchi, che nella totalità dei cerchi immaginari di queste lingue tra per esempio 10 cerchi, i cerchi neolatini corrispondono a sei, sette cerchi, che fanno da padrone nel lessico di queste lingue e che è comune a tutte queste lingue. Questi cerchi ovviamente sarebbero nell'ordine dei cerchi gli ultimi sette su dieci, e non i primi tre, che sono il lessico di base per le lingue anglofone e l'albanese. Tanto per fare un'idea tanti nomi astratti che finiscono con suffissi come -mento, zione, e suffissi aggettivali e verbi infiniti, che sono presenti nell'inglese, oltre alle lingue neolatine, perché questo lessico riguarda concetti di base di molti campi sociali e non sociali.

Le lingue sono degli organismi autonomi che funzionano senza complessi e i prestiti non provocano assolutamente perdite di originalità e appartenenza, non c'è la mentalità di lingue handicappate o inferiori, non ci sono discriminazioni in merito; ogni lingua è un patrimonio e monumento indiscusso del popolo, che lo parla e lo possiede.

Conclusioni; Ho fatto queste riflessioni per argomentare e capire che l'italiano, vivendo e operando nello stesso territorio dove è vissuto, ha operato, si è coltivato il latino non può tradire la sua origine e non può "contaminarsi" lessicalmente, se di contaminazione possiamo parlare, perché il fenomeno di dare e ricevere tra le lingue è un fenomeno molto linguistico e impregiudicato. Di questo l'albanese sa qualcosa, anzi molto, come somiglianza all'italiano dal punto di vista lessicale; è che molte volte non lo si vuole ammettere a voce alta.

\section{References}

John Lyons (1980). Manuale di Semantica, Laterza, Bari, Italia.

Luca Serianni e Pietro Trifone (1993-1994). Storia della lingua italiana, Einaudi, Torino, Italia.

P.Benigna, M.Berretta, M. Dardano, E.Magno Caldognetto, A. M. Mioni, B. Mortara Garavelli, P. Ramat, R.Simone, A.Sobrero, "Introduzione all'italiano contemporaneo. Le strutture. Editori Laterza 1993.

Tullio De Mauro (2008). "Storia linguistica dell'Italia unita", Laterza, Bari 2008.

Lo Zingarelli, (1994).Vocabolario della lingua italiana. Bologna: Zanichelli editore, S.p.A. Via Irnerio 34, 40126.

De Voto-Oli (2004). Dizionario della Lingua Italiana. Firenze: Felice Le Monnier, Edmund Le Monnier S.p.A. 
